\title{
Efflux pump-mediated drug resistance in Burkholderia
}

\author{
Nicole L. Podnecky ${ }^{1 \dagger}$, Katherine A. Rhodes ${ }^{1,2}$ and Herbert P. Schweizer ${ }^{1,2 *}$ \\ ${ }^{1}$ Department of Microbiology, Immunology and Pathology, College of Veterinary Medicine and Biological Sciences, Colorado \\ State University, Fort Collins, CO, USA, ${ }^{2}$ Department of Molecular Genetics and Microbiology, College of Medicine, \\ Emerging Pathogens Institute, Institute for Therapeutic Innovation, University of Florida, Gainesville, FL, USA
}

\section{OPEN ACCESS}

Edited by:

Keith Poole,

Queen's University, Canada

Reviewed by:

Veljo Kisand,

University of Tartu, Estonia

Giovanna Riccardi,

University of Pavia, Italy

${ }^{*}$ Correspondence:

Herbert P. Schweizer Department of Molecular Genetics and Microbiology, College of Medicine, Emerging Pathogens Institute, Institute for Therapeutic Innovation, University of Florida,

Gainesville, FL 32610, USA hschweizer@ufl.edu

${ }^{\dagger}$ Present address: Nicole L. Podnecky, Department of Pharmacy, Faculty of Health Sciences, UiT The Arctic University of Norway - Universitetet

Tromsø, 9037 Tromsø, Norway

Specialty section:

This article was submitted to Antimicrobials, Resistance and Chemotherapy, a section of the journal Frontiers in Microbiology

Received: 10 February 2015

Paper pending published: 06 March 2015

Accepted: 27 March 2015

Published: 14 April 2015

Citation:

Podnecky NL, Rhodes KA and Schweizer HP (2015) Efflux pump-mediated drug resistance in Burkholderia.

Front. Microbiol. 6:305. doi: 10.3389/fmicb.2015.00305
Several members of the genus Burkholderia are prominent pathogens. Infections caused by these bacteria are difficult to treat because of significant antibiotic resistance. Virtually all Burkholderia species are also resistant to polymyxin, prohibiting use of drugs like colistin that are available for treatment of infections caused by most other drug resistant Gram-negative bacteria. Despite clinical significance and antibiotic resistance of Burkholderia species, characterization of efflux pumps lags behind other non-enteric Gram-negative pathogens such as Acinetobacter baumannii and Pseudomonas aeruginosa. Although efflux pumps have been described in several Burkholderia species, they have been best studied in Burkholderia cenocepacia and B. pseudomallei. As in other non-enteric Gram-negatives, efflux pumps of the resistance nodulation cell division (RND) family are the clinically most significant efflux systems in these two species. Several efflux pumps were described in $B$. cenocepacia, which when expressed confer resistance to clinically significant antibiotics, including aminoglycosides, chloramphenicol, fluoroquinolones, and tetracyclines. Three RND pumps have been characterized in B. pseudomallei, two of which confer either intrinsic or acquired resistance to aminoglycosides, macrolides, chloramphenicol, fluoroquinolones, tetracyclines, trimethoprim, and in some instances trimethoprim+sulfamethoxazole. Several strains of the host-adapted B. mallei, a clone of $B$. pseudomallei, lack AmrAB-OprA, and are therefore aminoglycoside and macrolide susceptible. B. thailandensis is closely related to B. pseudomallei, but non-pathogenic to humans. Its pump repertoire and ensuing drug resistance profile parallels that of $B$. pseudomallei. An efflux pump in B. vietnamiensis plays a significant role in acquired aminoglycoside resistance. Summarily, efflux pumps are significant players in Burkholderia drug resistance.

Keywords: Burkholderia, antibiotics, resistance, efflux pump, adaptation

\section{The Genus Burkholderia}

The genus Burkholderia comprises metabolically diverse and adaptable Gram-negative bacteria that are able to thrive in different, often adversarial, environments. Their metabolic versatility and adaptability is in part due to the coding capacity provided by large (7-9 Mb) genomes consisting of several chromosomes and in some species, e.g., Burkholderia cenocepacia, plasmids (Holden et al., 2004, 2009; Agnoli et al., 2012). Many members of the genus are clinically significant pathogens 
with renowned virulence potential (Tegos et al., 2012) and drug resistance (Burns, 2007). In contrast to most other Gram-negative pathogens, Burkholderia species are intrinsically polymyxin resistant and therefore colistin cannot be used as drug of last resort (Loutet and Valvano, 2011). Despite clinical significance and recognized antibiotic resistance of Burkholderia species, characterization of efflux pumps lags significantly behind other nonenteric Gram-negative pathogens such as Acinetobacter baumannii and Pseudomonas aeruginosa (Nikaido and Pages, 2012). Many Burkholderia efflux systems have homologs in other Gramnegatives, including $A$. baumannii and $P$. aeruginosa, and it is now generally believed that the multidrug resistance exhibited by these opportunistic pathogens is largely attributable to the existence of similar efflux pumps in these organisms (Poole, 2001; McGowan, 2006). As with other Gram-negative bacteria, the relative roles that individual efflux pumps play in intrinsic or acquired antibiotic resistance in the respective Burkholderia species are in many instances difficult to discern for various reasons: (1) a subset of the pumps found in any organism usually exhibits a considerable degree of substrate promiscuity, i.e., they recognize and extrude chemically and structurally diverse compounds, which leads to similar multidrug resistance profiles; (2) many of the efflux systems are not expressed at significant levels in wild-type strains under laboratory conditions and there exists a significant knowledge gap regarding the environmental conditions under which efflux genes are expressed; and (3) well characterized clinical or laboratory isolates expressing or lacking the respective efflux pumps often do not exist or are difficult to obtain (Mima and Schweizer, 2010; Coenye et al., 2011; Biot et al., 2013; Buroni et al., 2014). In this review we will summarize the current state of knowledge of efflux pumps and their roles in antibiotic resistance in the genus Burkholderia, which have been characterized to various degrees in a few representative organisms.

\section{Burkholderia cepacia Complex}

The Burkholderia cepacia complex (BCC) currently comprises 17 closely related species (Mahenthiralingam et al., 2005; Vanlaere et al., 2009; Vandamme and Dawyndt, 2011). Some BCC members exhibit beneficial aspects such as use in biocontrol, a practice that has since been abandoned because of the risk of infection of compromised individuals (Kang et al., 1998). Many are opportunistic pathogens, being particularly problematic for cystic fibrosis patients and immune compromised individuals. $B$. cenocepacia and B. multivorans account for $85 \%$ of all BCC infections (Drevinek and Mahenthiralingam, 2010). BCC infections are difficult to treat because of intrinsic antibiotic resistance and persistence in the presence of antimicrobials (Golini et al., 2004; Peeters et al., 2009; Rajendran et al., 2010; Jassem et al., 2011). B. vietnamiensis belongs to the BCC group and sporadically infects cystic fibrosis patients (Jassem et al., 2011).

\section{Burkholderia pseudomallei}

Burkholderia pseudomallei is a saprophyte and opportunistic pathogen endemic to tropical and subtropical regions of the world, and recent studies suggest that is more widespread than previously thought (Wiersinga et al., 2006, 2012, 2015; Currie et al., 2010). Since the U.S. anthrax attacks in 2001 the bacterium has received increasing attention because of its biothreat potential (Cheng et al., 2005), a history dating back to its use with malicious intent in a Sherlock Holmes short story (Vora, 2002). In the U.S. it is a strictly regulated Tier 1 select agent, which must be handled in approved biosafety level 3 (BSL-3) laboratories. The bacterium is the etiologic agent of melioidosis, a difficultto-treat multifacteted disease (Wiersinga et al., 2006, 2012). The disease affects at-risk patients, including those suffering from cystic fibrosis (Schulin and Steinmetz, 2001; Holland et al., 2002), non-cystic fibrosis bronchiectasis (Price et al., 2013), and diabetes (Simpson et al., 2003). B. pseudomallei infections are recalcitrant to antibiotic therapy because of the bacterium's intrinsic resistance due to expression of resistance determinants such as $\beta$-lactamase and efflux pumps, as well as contributing factors such as both intracellular and biofilm lifestyles (Schweizer, 2012b).

\section{Burkholderia mallei}

Burkholderia mallei is an obligate zoonotic pathogen and the etiologic agent of glanders, which has been used as a bioweapon (Cheng et al., 2005; Whitlock et al., 2007). This bacterium likely diverged from $B$. pseudomallei upon introduction into an animal host approximately 3.5 million years ago (Losada et al., 2010; Song et al., 2010). The ensuing in-host evolution through massive expansion of insertion (IS) elements, IS-mediated gene deletion, and genome rearrangement, and prophage elimination is likely also responsible for the generally increased antibiotic susceptibility of B. mallei when compared to B. pseudomallei, presumably due to inactivation of resistance determinants (Nierman et al., 2004).

\section{Burkholderia thailandensis}

Burkholderia thailandensis is closely related to B. pseudomallei (Brett et al., 1998). Although B. thailandensis has sporadically been shown to cause human disease (Glass et al., 2006), it is generally considered non-pathogenic and has often been used as a surrogate for antimicrobial compound and vaccine efficacy studies because the bacterium can be handled at BSL-2. Some strains are more closely related to $B$. pseudomallei than others. For instance, unlike the widely used $B$. thailandensis prototype strain E264 (Brett et al., 1998), strain E555 expresses the same capsular polysaccharide as B. pseudomallei (Sim et al., 2010). Since capsular polysaccharide is a potent immunogen this similarity was exploited in a vaccine study, which showed that immunization with live cells of this avirulent strain protects mice from challenge with a virulent B. pseudomallei strain (Scott et al., 2013).

\section{Burkholderia cenocepacia}

\section{Efflux Pumps and Drug Resistance}

Early reports provided mostly indirect evidence that $B$. cenocepacia efflux pumps play a role in drug efflux. The synergy between reduced outer membrane permeability and efflux was cited as a common theme of the increased resistance that nonfermenting Gram-negative bacteria like A. baumannii, $P$. aeruginosa, and B. (ceno)cepacia display (Hancock, 1998). An analysis 
of the DsbA-DsbB disulfide bond formation system revealed that $d s b A$ and $d s b B$ mutation resulted increased susceptibilities to a variety of antibiotics (Hayashi et al., 2000). This led to the conclusion that the DsbA-DsbB system might be involved in the formation of a multidrug resistance system. Another early report described an outer membrane lipoprotein involved in multiple antibiotic (chloramphenicol, trimethoprim, and ciprofloxacin) resistance (Burns et al., 1996). This protein, OpcM, is the outer membrane channel of an efflux pump of the resistance nodulation cell division (RND) family that was subsequently named CeoAB-OpcM, which was shown to be inducible by salicylate and chloramphenicol (Nair et al., 2004). Efflux was also shown early on to play a role in fluoroquinolone resistance (Zhang et al., 2001).

Genome analysis and homology searches led to identification of an additional 14 open reading frames encoding putative components RND family efflux pumps (Guglierame et al., 2006). A summary of pertinent features of at least partially characterized B. cenocepacia RND efflux pumps and their relationships to RND systems in other Burkholderia species is presented in Table $\mathbf{1 .}$
Expression of one of these, orf2, in Escherichia coli conferred resistance to several antibiotics (Guglierame et al., 2006). The roles of several RND transporters - RND-1, RND-3, and RND4 - in intrinsic B. cenocepacia drug resistance was subsequently assessed by mutational analyses, which showed that RND-3 and RND-4, but not RND-1, contribute to B. cenocepacia's intrinsic resistance to antibiotics and other inhibitory compounds (Buroni et al., 2009). A subsequent study comparing RND-4 and RND9 single and double mutants confirmed the role that RND-4 plays in B. cenocepacia's antibiotic resistance and also showed that RND-9 contributed only marginally to resistance (Bazzini et al., 2011b). Completion of the strain J2315 genome sequence showed that it encodes 16 RND efflux systems, which provides evidence for the biological relevance of these transporters in this bacterium and also enables global analyses of RND pump expression (Holden et al., 2009; Perrin et al., 2010; Bazzini et al., 2011a; Buroni et al., 2014). For example, deletion of the 16 putative RND operons from B. cenocepacia strain J2315 showed that these pumps play differential roles in the drug resistance of sessile (biofilm) and planktonic cells. These studies revealed that: (1)

TABLE 1 | Partially characterized resistance nodulation cell division (RND) efflux pumps in Burkholderia species.

\begin{tabular}{|c|c|c|c|c|c|}
\hline Species & Efflux pump & Gene names & Gene annotation & Major substrates & Reference \\
\hline \multicolumn{6}{|c|}{$\begin{array}{l}\text { Burkholderia } \\
\text { cenocepacia }\end{array}$} \\
\hline & RND-1 & NA & BCAS0591-BCAS0593 & Non-detectable & Buroni et al. (2009) \\
\hline & RND-3 & $\mathrm{NA}^{1}$ & BCAL1674-BCAL1676 & $\begin{array}{l}\text { Nalidixic acid, ciprofloxacin, } \\
\text { tobramycin, chlorhexidine }{ }^{3}\end{array}$ & $\begin{array}{l}\text { Buroni et al. (2009, 2014), } \\
\text { Coenye et al. (2011) }\end{array}$ \\
\hline & RND-4 & $N A^{2}$ & BCAL2820-BCAL2822 & $\begin{array}{l}\text { Aztreonam, chloramphenicol, } \\
\text { fluoroquinolones, tobramycin }\end{array}$ & Bazzini et al. (2011b) \\
\hline & RND-8 & NA & BCAM0925-BCAM0927 & Tobramycin ${ }^{3}$ & Buroni et al. (2014) \\
\hline & RND-9 & NA & BCAM1945-BCAM1947 & Tobramycin $^{3}$, chlorhexidine ${ }^{3}$ & $\begin{array}{l}\text { Coenye et al. (2011), Buroni } \\
\text { et al. (2014) }\end{array}$ \\
\hline & RND-10 & сеоAB-орсM $M^{4}$ & BCAM2551-BCAM2549 & $\begin{array}{l}\text { Chloramphenicol, fluoroquinolones, } \\
\text { trimethoprim }\end{array}$ & Nair et al. (2004) \\
\hline \multicolumn{6}{|c|}{ B. pseudomallei } \\
\hline & AmrAB-OprA & amrAB-oprA & BPSL1804-BPSL1802 & $\begin{array}{l}\text { Aminoglycosides, macrolides, } \\
\text { cethromycin }\end{array}$ & $\begin{array}{l}\text { Moore et al. (1999), Mima et al. } \\
\text { (2011) }\end{array}$ \\
\hline & BpeAB-OprB & bpeAB-oprB & BPSL0814-BPSL0816 & $\begin{array}{l}\text { Chloramphenicol, fluoroquinolones, } \\
\text { macrolides, tetracyclines }{ }^{5}\end{array}$ & $\begin{array}{l}\text { Chan et al. (2004), Mima and } \\
\text { Schweizer (2010) }\end{array}$ \\
\hline & BpeEF-OprC & bpeEF-oprC & BPSS0292-BPSS0294 & $\begin{array}{l}\text { Chloramphenicol, fluoroquinolones, } \\
\text { tetracyclines, trimethoprim }{ }^{6}\end{array}$ & $\begin{array}{l}\text { Kumar et al. (2006), Schweizer } \\
(2012 a, b)\end{array}$ \\
\hline \multicolumn{6}{|c|}{ B. thailandensis } \\
\hline & AmrAB-OprA & amrAB-oprA & BTH_I2445-BTH_I2443 & $\begin{array}{l}\text { Aminoglycosides, macrolides, } \\
\text { tetracyclines }\end{array}$ & Biot et al. (2013) \\
\hline & BpeAB-OprB & bpeAB-oprB & BTH_I0680-BTH_I0682 & Tetracyclines & Biot et al. (2013) \\
\hline & BpeEF-OprC & bpeEF-oprC & BTH_II2106-BTH_II2104 & $\begin{array}{l}\text { Chloramphenicol, fluoroquinolones, } \\
\text { tetracyclines, } \\
\text { trimethoprim/sulfamethoxazole }\end{array}$ & Biot et al. (2011, 2013) \\
\hline \multicolumn{6}{|c|}{ B. vietnamiensis } \\
\hline & AmrAB-OprM & amrAB-oprM & $\begin{array}{l}\text { Bcep1808_1574- } \\
\text { Bcep1808_1576 }\end{array}$ & Aminoglycosides & Jassem et al. (2014) \\
\hline
\end{tabular}

NA, not applicable

${ }^{1}$ Corresponds to B. pseudomallei amrAB-oprA

${ }^{2}$ Corresponds to B. pseudomallei bpeAB-oprB

${ }^{3}$ Sessile (biofilm grown) cells only

${ }^{4}$ Corresponds to B. pseudomallei bpeEF-oprC

${ }^{5}$ Low-level resistance in de-repressed ( $\triangle$ bpeR) strains

${ }^{6}$ High-level resistance in regulatory mutants, e.g., bpeT carboxy-terminal mutations 
RND-3 and RND-4 play important roles in resistance to various antibiotics, including ciprofloxacin and tobramycin, in planktonic populations; (2) RND-3, RND-8, and RND-9 protect from the antimicrobial effects of tobramycin in biofilm cells; and (3) RND-8 and RND-9 do not play a role in ciprofloxacin resistance (Buroni et al., 2014). An emerging theme from these studies is that RND-3 seems to play a major role in B. cenocepacia's intrinsic drug resistance. It was suggested that mutations in the RND3 regulator-encoding gene may be responsible for this pump's prevalent overexpression and accompanying high-level antibiotic resistance in clinical BCC isolates (Tseng et al., 2014).

Studies aimed at assessing chlorhexidine mechanisms in $B$. cenocepacia J2315 confirmed the differential roles that RND pumps play in biofilm versus planktonically grown cells. RND4 contributed to chlorhexidine resistance in planktonic cells, whereas RND-3 and RND-9 played a role in chlorhexidine resistance in sessile cells (Coenye et al., 2011). Mutational analyses of 2-thiopyridine resistant mutants showed that RND-4 confers resistance to an anti-tubercular 2-thiopyridine derivative (Scoffone et al., 2014). The involvement of efflux pumps in tigecycline resistance was inferred from the potentiating effects of the efflux pump inhibitor (EPI) MC-207,110 on tigecycline's anti- $B$. cenocepacia activity (Rajendran et al., 2010).

Efflux pumps belonging to other families may also contribute to $B$. cenocepacia's drug resistance. Experiments with an immunodominant antigen in cystic fibrosis patients infected with $B$. cenocepacia identified a drug efflux pump, BcrA, which is a member of the major facilitator superfamily (MFS). It was shown to confer tetracycline and nalidixic acid resistance when expressed in E. coli (Wigfield et al., 2002). Upregulation of an efflux pump resulted in resistance to the phosphonic acid antibiotic fosfidomycin (Messiaen et al., 2011). This pump is a homolog of Fsr, a member of the MFS, which was previously shown to confer fosmidomycin resistance on E. coli (Fujisaki et al., 1996; Nishino and Yamaguchi, 2001).

\section{Other Functions of B. cenocepacia RND Efflux Pumps}

As with other Gram-negative bacteria, the function of B. cenocepacia efflux pumps extends beyond antibiotic resistance. In $B$. cenocepacia, these systems are involved in modulation of virulence-associated traits such as quorum sensing, biofilm formation, chemotaxis, and motility, as well as general physiological functions (Buroni et al., 2009; Bazzini et al., 2011a,b). A proteomic analysis of the effects of RND-4 gene deletion revealed about 70 differentially expressed proteins, most of which were associated with cellular functions other than drug resistance. This suggests that RND-4 plays a more general role in B. cenocepacia's biology (Gamberi et al., 2013). Aside from the key role that efflux, especially RND-mediated efflux, plays in adaptation to antibiotic exposure (Bazzini et al., 2011b; Sass et al., 2011; Tseng et al., 2014), survival of Burkholderia species in various niche environments and accompanying conditions, e.g., the cystic fibrosis airways (Mira et al., 2011), marine habitats (Maravic et al., 2012), oxygen levels (Hemsley et al., 2014), exposure to noxious chemicals (Rushton et al., 2013), and other ecological niches (Liu et al.,
2015), involves to various degrees changes in efflux pump expression.

\section{Burkholderia pseudomallei}

\section{Efflux Pumps and Drug Resistance}

Initially, the presence of genomic DNA sequences in B. pseudomallei that hybridize with the multidrug resistance efflux gene oprM of $P$. aeruginosa was interpreted as evidence that efflux-mediated multidrug efflux systems may also be present in B. pseudomallei (Bianco et al., 1997). A recent survey of documented $B$. pseudomallei antibiotic resistance mechanisms indeed showed that efflux is the dominant mechanism affecting most classes of antibiotics (Schweizer, 2012b). Sequenced $B$. pseudomallei genomes encode numerous efflux systems, but as with other non-enteric bacteria only RND pumps have to date been shown to confer resistance to clinically significantly antibiotics. The K96243 and other B. pseudomallei genomes encode at least 10 RND systems, seven of which are encoded by chromosome 1 and three by chromosome 2 (Holden et al., 2004; Figure 1A). Although RND operon distribution is conserved amongst diverse $B$. pseudomallei strains, locations on the respective chromosomes may vary because of chromosome rearrangements. Bioinformatic analyses indicate that not all of the RND operons encode drug efflux pumps. For instance, one system seems to encode components of a general secretion ( $\mathrm{Sec}$ ) system. Although the presence of many RND systems can be detected in clinical and environmental isolates at the transcriptional (Kumar et al., 2008) and protein level (Schell et al., 2011), this expression is not necessarily linked to increased drug resistance. Meaningful studies to address their function are complicated because isogenetic progenitor and/or comparator strains are generally not available. Further hindering efflux pump characterization are select agent guidelines, which restrict certain methods, such as selection of spontaneous drug resistant mutants that may display altered efflux expression profiles. These investigations are now facilitated by the availability of several $B$. pseudomallei strains, for instance Bp82 (Propst et al., 2010), which are excluded from select agent rulings. To date three RND drug efflux pumps - AmrAB-OprA, BpeAB-OprB, and BpeEFOprC - have been characterized in some detail (Figure 1B). There is evidence that small molecule compounds such as MC207,110, phenothiazine antipsychotics, and antihistaminic drugs like promazine can be used to potentiate antibiotic efficacy, primarily by inhibition of RND efflux pumps (Chan et al., 2007b).

\section{AmrAB-OprA}

The AmrAB-OprA efflux pump was the first efflux pump described in B. pseudomallei (Moore et al., 1999). It is responsible for this organism's high-level intrinsic aminoglycoside and macrolide resistance (Moore et al., 1999; Viktorov et al., 2008). Rare $(\sim 1$ in 1,000$)$ naturally occuring aminoglycoside susceptible $B$. pseudomallei isolates have previously been identified (Trunck et al., 2009; Podin et al., 2014). They do not 


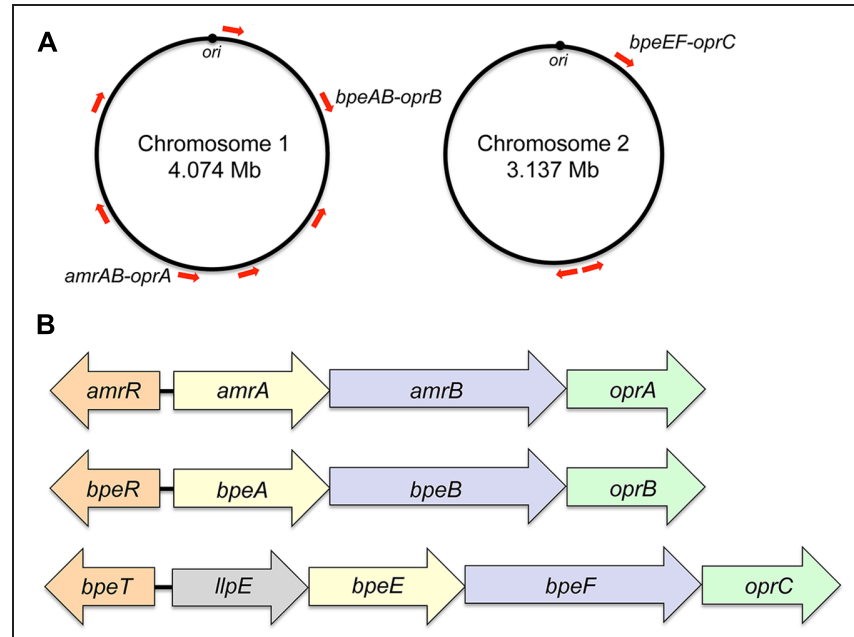

FIGURE 1 | Genomic location and operon organization of Burkholderia pseudomallei resistance nodulation cell division (RND) efflux pumps.

(A) Chromosomal locations of RND efflux operons in strain K96243. Red arrows indicate the approximate locations and transcriptional orientations of RND operons on chromosomes 1 and 2. Operons encoding characterized efflux pumps are labeled. Although RND operon distribution is conserved amongst diverse $B$. pseudomallei strains, locations on the respective chromosomes may vary because of chromosome rearrangements. The origins of replication (ori) in the two strain K96243 chromosomes are marked. Their respective locations on the chromosomes of other strains may vary because of chromosome rearrangements. (B) Transcriptional organization of characterized RND operons. The genes encoding the membrane fusion proteins (amrA, bpeA, bpeE), RND transporter (amrB, bpeB, bpeF) and outer membrane protein channel (oprA, oprB, oprC) are color-coded. The bpeE-bpeF-oprC genes are in the same operon as IlpE, which is annotated as a putative lipase/carboxyl esterase. LIpE is not required for BpeEF-OprC efflux pump function. Orthologs of $L \mathrm{p} E$ are present in all annotated operons that encode bpeE-bpeF-oprC orthologs. The amrR and bpeR genes encode repressors of amrAB-oprA and bpeAB-oprB, respectively. The bpe $T$ gene encodes a LysR-type transcriptional regulator of IlpE-bpeE-bpeF-oprC.

express the AmrAB-OprA pump either due to regulatory mutations (Trunck et al., 2009), point mutations affecting the AmrB RND transporter amino acid sequence (Podin et al., 2014), or because the entire amrAB-oprM operon is missing due to a genomic deletion (Trunck et al., 2009). Although the AmrAB-OprA efflux pump is expressed in prototype strains, exposure to antimicrobials can select for unknown mutations that cause its over-expression resulting in increased resistance. For instance, prototype strain $1026 \mathrm{~b}$ is moderately susceptible [minimal inhibitory concentration (MIC) $4-8 \mu \mathrm{g} / \mathrm{mL}$ ] to the ketolide cethromycin and exposure to this compound readily selects for highly resistant ( $\mathrm{MIC}>128 \mu \mathrm{g} / \mathrm{mL}$ ) derivatives due to AmrAB-OprA over-expression (Mima et al., 2011). To date, AmrAB-OprA expression is the sole reported aminoglycoside and macrolide resistance mechanism observed in B. pseudomallei.

AmrAB is closely related to $P$. aeruginosa MexXY, which is expressed in some aminoglycoside resistant mutants and together with OprM constitutes a functional effux pump (Mine et al., 1999; Sobel et al., 2003; Morita et al., 2012). MexXY associates with the mexAB-oprM encoded OprM outer channel protein because the
mexXY operons of most $P$. aeruginosa strains do not encode a cognate outer mebrane channel protein. However, some strains, e.g., $P$. aeruginosa PA7, encode a mexAB-oprA operon akin and functionally equivalent to $B$. pseudomallei amrAB-oprA (Morita et al., 2012).

\section{BpeAB-OprB}

The BpeAB-OprB efflux pump was first described in strain KHW from Singapore (Chan et al., 2004) and subsequently characterized in Thai strain 1026b (Mima and Schweizer, 2010). It is not significantly expressed in wild-type strains. BpeABOprB expression is regulated by BpeR and $b p e R$ mutants exhibit low-level chloramphenicol, fluoroquinolone, tetracycline, and macrolide resistance (Chan et al., 2004; Chan and Chua, 2005; Mima and Schweizer, 2010). Although the original studies with strain KHW indicated a role of BpeAB-OprB in aminoglycoside resistance (Chan et al., 2004), these results could not be confirmed with strain 1026b (Mima and Schweizer, 2010). At present, the observed differences in BpeAB-OprB substrate spectrum between strains KHW and 1026b are not understood. Because of the low levels of resistance bestowed by BpeAB-OprB and naturally occuring antibiotic resistant $\mathrm{Bpe} \mathrm{AB}-\mathrm{OprB}$ over-expressing mutants have yet to be identified, the clinical significance of this pump remains unclear.

Although BpeAB-OprB is related to P. aeruginosa MexABOprM (Poole et al., 1993; Li et al., 1995; Mima and Schweizer, 2010), the respective features are quite divergent. While $P$. aeruginosa MexAB-OprM is widely expressed and responsible for this bacterium's intrinsic resistance to numerous antibacterial compounds (Poole et al., 1993; Li et al., 1995; Poole, 2001), B. pseudomallei BpeAB-OprB is not widely expressed and does seem to play only a minor role in this bacterium's resistance to antimicrobials.

\section{BpeEF-OprC}

BpeEF-OprC was first identified as a chloramphenicol and trimethoprim efflux pump by expression in an efflux-compromised $P$. aeruginosa strain (Kumar et al., 2006). This pump is not expressed in B. pseudomallei wildtype strains, but only regulatory mutants. For instance, it is constitutively expressed in naturally occuring bpeT mutants (Hayden et al., 2012; Figure 2). When expressed, BpeEF-OprC confers high-level resistance to chloramphenicol, fluoroquinolones, tetracyclines, and trimethoprim. It is responsible for widespread trimethoprim resistance in clinical and environmental B. pseudomallei isolates (Podnecky et al., 2013). Pump expression is inducible by some pump substrates, which when present at sub MIC levels may lead to cross-resistance with other pump substrates (Schweizer, 2012a).

BpeEF-OprC is related to $P$. aeruginosa MexEF-OprN (Koehler et al., 1997), which shares properties such as substrate profiles and selection of pump-expressing regulatory mutants by chloramphenicol as previously demonstrated with both $P$. aeruginosa MexEF-OprN (Koehler et al., 1997) and B. thailandensis BpeEF-OprC (Biot et al., 2011). 


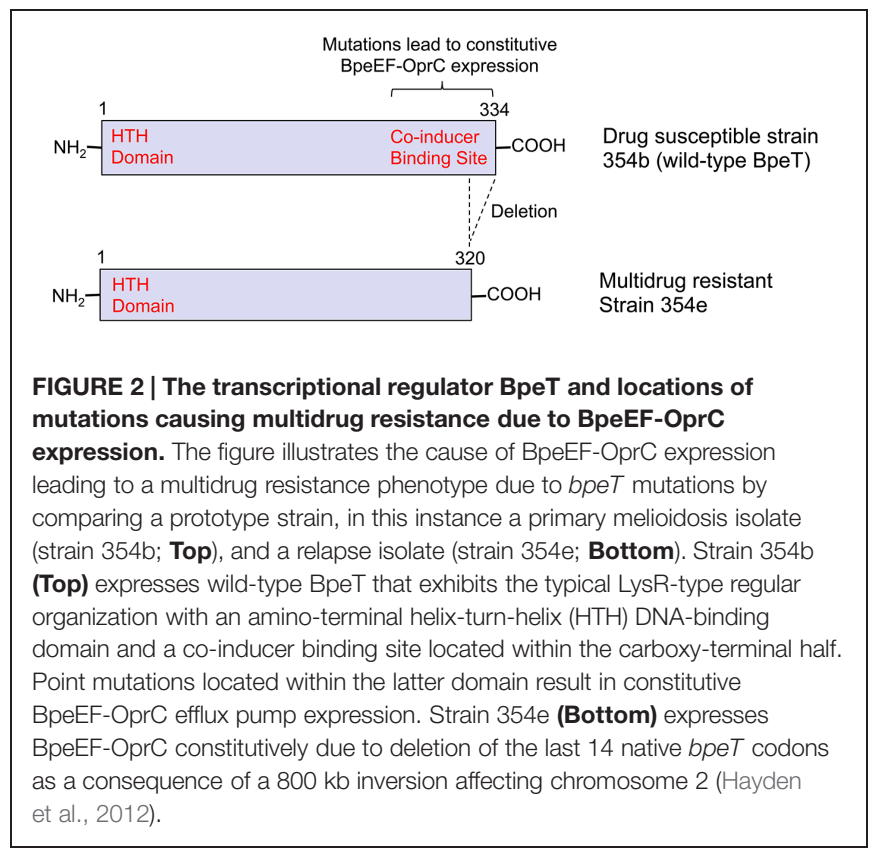

\section{Other Functions of B. pseudomallei Efflux Pumps}

Quorum sensing is an important determinant of virulence factor regulation in bacteria. Numerous studies with $P$. aeruginosa indicate the involvement of several RND pumps in quorum sensing and thus several virulence traits by being involved in transport of cell-to-cell signaling molecules and their inhibitors (Evans et al., 1998; Pearson et al., 1999; Koehler et al., 2001; Aendekerk et al., 2005; Hirakata et al., 2009; Tian et al., 2009). At least one efflux pump regulator, MexT, modulates expression of virulence factors, albeit independent of the function of the MexEF-OprN efflux pump whose expression it regulates (Tian et al., 2009). A $P$. aeruginosa MexAB-OprM deletion strain is also compromised in its ability to invade Madin-Darby canine kidney (MDCK) cells (Hirakata et al., 2009). Based on these findings with P. aeruginosa, several studies with $B$. pseudomallei explored the effects of efflux on quorum sensing and virulence. Studies with strain KHW showed that the BpeAB-OprB efflux pump was required: (1) for the secretion of the acyl homoserine lactones produced by this strains quorum-sensing systems (Chan et al., 2007a); and (2) secretion of several virulence-associated determinants, including siderophore and biofilm formation (Chan and Chua, 2005), but these observations could not be confirmed with strain 1026b (Mima and Schweizer, 2010). Cell invasion of and cytotoxicity toward human A549 lung epithelial and THP-1 macrophage cell were signifianctly reduced in a KHW BpeAB-deficient strain (Chan and Chua, 2005). Adherence to A549 cells and virulence in the $\mathrm{BALB} / \mathrm{c}$ mouse intranasal infection model were not affected in the AmrAB-OprA deficient Bp340 mutant, a derivative of strain 1026b (Campos et al., 2013). BALB/c mouse intranasal infection studies also showed that in addition to AmrAB-OprA, BpeAB-OprB, and BpeEF-OprC were not required for virulence (Propst, 2011; Schweizer, 2012a). The AmrAB-OprA efflux pump is also not required for efficient killing of Caenorhabditis elegans by B. pseudomallei (O'Quinn et al., 2001). The BpeABOprB pump has been implicated in being involved in spermidine homeostasis in strain KHW with exogenous spermidine and $\mathrm{N}$ acetylspermidine activating bpeA transcription (Chan and Chua, 2010).

\section{Efflux Pumps in other Burkholderia Species}

\section{Burkholderia mallei}

In part due to ongoing in host evolution of this obligate pathogen, $B$. mallei is generally more susceptible to antimicrobials than its progenitor $B$. pseudomallei. For instance, many $B$. mallei strains are susceptible to aminoglycosides. In the ATCC 23344 prototype strain this susceptibility results from a $50 \mathrm{~kb}$ chromosomal deletion encompassing the amrAB-oprA operon (Nierman et al., 2004). Strains NCTC10229 and NCTC10247 are likely aminoglycoside susceptible because only remnants of the amrAB-oprA operon are present (Winsor et al., 2008). Genes and operons encoding other efflux pumps, including BpeAB-OprB and BpeEF-OprC, are present but whether they encode functional efflux systems remains remains to be established.

\section{Burkholderia thailandensis}

One study indicated the presence of an MFS efflux pump, with an associated regulatory protein of the multiple antibiotic resistance regulator (MarR) family (Grove, 2010). However, expression of the efflux pump was only responsive to urate, xanthine, and hypoxanthine and thus the significance of this transporter in $B$. thailandensis' antibiotic resistance, if any, is unclear.

In contrast, the contributions of RND pumps to this bacterium's antibiotic resistance have been established. It was shown that chloramphenicol exposure selects for expression of an RND efflux pump, BpeEF-OprC, that also extrudes fluoroquinolones, tetracycline, and trimethoprim (Biot et al., 2011). Doxycycline selection resulted in mutants that either over-expressed AmrAB-OprA or BpeEF-OprC, and exhibited the multidrug resistance profiles associated with expression of these efflux pumps (Biot et al., 2013). Mutational analysis of these mutants suggested that BpeAB-OprB could at least partially substitute for absence of either AmrAB-OprA or BpeEF-OprC. Unlike other Gram-negative bacteria, cell envelope properties, efflux pump repertoire, and resulting drug resistance profile make $B$. thailandensis suitable as a $B$. pseudomallei BSL-2 surrogate for drug efficacy studies (Schweizer, 2012c).

\section{Burkholderia vietnamiensis}

Transposon mutagenesis studies aimed at identification of polymyxin B susceptible mutants identified a gene encoding a NorM multidrug efflux protein (Fehlner-Gardiner and Valvano, 2002). While nor $M$ expression in an E. coli acr $A B$ deletion mutant complemented its norfloxacin susceptibility, its inactivation in $B$. vietnamiensis only affected susceptibility to polymyxin but not other antibiotics. 
Unlike other Burkholderia species, including most BCC members, the majority of environmental and clinical B. vietnamiensis isolates are aminoglycoside susceptible (Jassem et al., 2011). Aminoglycoside resistance as a result of chronic infection or in vitro exposure to aminoglycosides is the result of the AmrABOprM efflux pump, which is most likely a homolog of $B$. pseudomallei and B. thailandensis AmrAB-OprA (Jassem et al., 2011, 2014). Of note is the observation that efflux pump expression in mutants that acquired resistance during infection was due to missense mutations in the $a m r A B$-oprM regulator $a m r R$, but not those mutants derived from antibiotic pressure in vivo (Jassem et al., 2014).

\section{Burkholderia Efflux Pump Mutants as Experimental Tools}

The high-level intrinsic antibiotic resistance of many Burkholderia species complicates their genetic manipulation, use in studies of intracellular bacteria with the aminoglycoside protection assay, and drug efficacy studies. It has been shown that efflux-compromised mutants of B. cenocepacia and B. pseudomallei greatly facilitate genetic manipulation of these species, as well as cell invasion studies using the aminoglycoside protection assay (Dubarry et al., 2010; Hamad et al., 2010; Campos et al., 2013). Efflux-compromised strains of B. thailandensis and B. pseudomallei strains have also proved useful for study of the efflux propensity of novel antimicrobial compounds (Liu et al., 2011; Mima et al., 2011; Teng et al., 2013; Cummings et al., 2014).

\section{Conclusion}

Burkholderia species are well adapted to life in diverse, often adversarial, environments including those containing antimicrobials. This adaptation is facilitated by large genomes that bestow on the bacteria the ability to either degrade or expel noxious chemicals. As a result, opportunistic infections by pathogenic members of this species are difficult to treat because of intrinsic antibiotic resistance and persistence in the presence of antimicrobials. Resistance is in large part attributable to efflux pump expression, mostly members of the RND family. While the last decade has seen significant progress in study of drug efflux in Burkholderia species, progress still lags significantly behind other opportunistic pathogens, e.g., P. aeruginosa and A. baumannii, where efflux pumps also play significant roles in intrinsic and acquired drug resistance.

There are some unique aspects of efflux systems in Burkholderia species that are without parallel and studies of these

\section{References}

Aendekerk, S., Diggle, S. P., Song, Z., Hoiby, N., Cornelis, P., Williams, P., et al. (2005). The MexGHI-OpmD multidrug efflux pump controls growth, antibiotic susceptibility and virulence in Pseudomonas aeruginosa via 4-quinolone-dependent cell-to-cell communication. Microbiology 151, 1113-1125. doi: 10.1099/mic.0.2 7631-0 may shed light on unique physiological functions of efflux pumps in these organisms. For instance, the first gene in the bpeEF$\operatorname{opr} C$ operon, $l l p E$, is co-transcribed with the genes encoding the BpeEF-OprC efflux pump components (Nair et al., 2004, 2005). Its deletion neither affects efflux pump function nor specificity for known antibiotic substrates. It is highly conserved throughout the Burkholderia genus and found in all sequenced genomes (Winsor et al., 2008). Based on homology, LlpE probably is an enzyme of the alpha/beta hydrolase family, recently annotated as a putative lipase/carboxyl esterase, and its conservation throughout the genus suggests an adaptation or survival benefit in a niche environment. The unique association of this enzyme with BpeEFOprC and its role in Burkholderia biology warrant further studies of this enzyme.

When reviewing the $B$. cenocepacia efflux pump literature it becomes evident that efflux pump nomenclature in this species, especially that of the RND family is non-uniform and confusing (for instance CeoAB-OpcM, RND-1 to RND-16, BCA gene names, Mex1, orf, etc.), which makes comparisons with other Gram-negative bacteria unnecessarily cumbersome. As with other Gram-negative bacteria, the nomenclature initiated in B. cenocepacia by Dr. Jane Burns' laboratory in the early 2000s, i.e., CeoAB-OpcM (Nair et al., 2004), would make the most sense and it is a pity that it was not followed in subsequent studies.

\section{Author Contributions}

NP, KR, and HS contributed to all aspects of the work, including, but not limited to, conception and design, acquisition and analysis of data, writing the manuscript, and final approval of the version to be published.

\section{Acknowledgments}

The authors acknowledge the contributions of several talented graduate students (Katie Propst, Kyoung-Hee Choi, Lily Trunck, Carolina Lopez) and postdocs (Takehiko Mima, Ayush Kumar, Nawarat Somprasong) to efflux pump research performed in the Schweizer laboratory. We acknowledge Dr. Hillary Hayden from the University of Washington for providing sequence information on B. pseudomallei strains 354b and 354e. Work in the HPS laboratory was supported by grant AI065357 from the National Institute of Allergy and Infectious Diseases, National Institutes of Health, and contract HDTRA1-08-C-0049 from the United States Defense Threat Reduction Agency.

Agnoli, K., Schwager, S., Uehlinger, S., Vergunst, A., Viteri, D. F., Nguyen, D. T., et al. (2012). Exposing the third chromosome of Burkholderia cepacia complex strains as a virulence plasmid. Mol. Microbiol. 83, 362-378. doi: 10.1111/j.13652958.2011.07937.x

Bazzini, S., Udine, C., and Riccardi, G. (2011a). Molecular approaches to pathogenesis study of Burkholderia cenocepacia, an important cystic fibrosis opportunistic bacterium. Appl. Microbiol. Biotechnol. 92, 887-895. doi: 10.1007/s00253011-3616-5 
Bazzini, S., Udine, C., Sass, A., Pasca, M. R., Longo, F., Emiliani, G., et al. (2011b). Deciphering the role of RND efflux transporters in Burkholderia cenocepacia. PLOS ONE 6:e18902. doi: 10.1371/journal.pone.0018902

Bianco, N., Neshat, S., and Poole, K. (1997). Conservation of the multidrug resistance efflux gene oprM in Pseudomonas aeruginosa. Antimicrob. Agents Chemother. 41, 853-856.

Biot, F. V., Lopez, M. M., Poyot, T., Neulat-Ripoll, F., Lignon, S., Caclard, A., et al. (2013). Interplay between three RND efflux pumps in doxycycline-selected strains of Burkholderia thailandensis. PLoS ONE 8:e84068. doi: 10.1371/journal.pone. 0084068

Biot, F. V., Valade, E., Garnotel, E., Chevalier, J., Villard, C., Thibault, F. M., et al. (2011). Involvement of the efflux pumps in chloramphenicol selected strains of Burkholderia thailandensis: proteomic and mechanistic evidence. PLOS ONE 6:e16892. doi: 10.1371/journal.pone.0016892

Brett, P. J., Deshazer, D., and Woods, D. E. (1998). Burkholderia thailandensis sp. nov., a Burkholderia pseudomallei-like species. Int. J. Syst. Bacteriol. 48, 317-320. doi: 10.1099/00207713-48-1-317

Burns, J. (2007). "Antibiotic resistance of Burkholderia spp.," in Burkholderia Molecular Microbiology and Genomics, eds T. Coenye and P. Vandamme (Norfolk: Horizon Bioscience), 81-91.

Burns, J. L., Wadsworth, C. D., Barry, J. J., and Goodall, C. P. (1996). Nucleotide sequence analysis of a gene from Burkholderia (Pseudomonas) cepacia encoding an outer membrane lipoprotein involved in multiple antibiotic resistance. Antimicrob. Agents Chemother. 40, 307-313.

Buroni, S., Matthijs, N., Spadaro, F., Van Acker, H., Scoffone, V. C., Pasca, M. R., et al. (2014). Differential roles of RND efflux pumps in antimicrobial drug resistance of sessile and planktonic Burkholderia cenocepacia cells. Antimicrob. Agents Chemother. 58, 7424-7429. doi: 10.1128/AAC.03800-14

Buroni, S., Pasca, M. R., Flannagan, R. S., Bazzini, S., Milano, A., Bertani, I., et al. (2009). Assessment of three Resistance-Nodulation-Cell Division drug efflux transporters of Burkholderia cenocepacia in intrinsic antibiotic resistance. BMC Microbiol. 9:200. doi: 10.1186/1471-2180-9-200

Campos, C. G., Borst, L., and Cotter, P. A. (2013). Characterization of BcaA, a putative classical autotransporter protein in Burkholderia pseudomallei. Infect. Immun. 81, 1121-1128. doi: 10.1128/IAI.01453-12

Chan, Y. Y., Bian, H. S., Tan, T. M., Mattmann, M. E., Geske, G. D., Igarashi, J., et al. (2007a). Control of quorum sensing by a Burkholderia pseudomallei multidrug efflux pump. J. Bacteriol. 189, 4320-4324. doi: 10.1128/JB.00 003-07

Chan, Y. Y., Ong, Y. M., and Chua, K. L. (2007b). Synergistic interaction between phenothiazines and antimicrobial agents against Burkholderia pseudomallei. Antimicrob. Agents Chemother. 51, 623-630. doi: 10.1128/AAC.01 033-06

Chan, Y. Y., and Chua, K. L. (2005). The Burkholderia pseudomallei BpeABOprB efflux pump: expression and impact on quorum sensing and virulence. J. Bacteriol. 187, 4707-4719. doi: 10.1128/JB.187.14.4707-4719.2005

Chan, Y. Y., and Chua, K. L. (2010). Growth-related changes in intracellular spermidine and its effect on efflux pump expression and quorum sensing in Burkholderia pseudomallei. Microbiology 156, 1144-1154. doi: 10.1099/mic.0.032888-0

Chan, Y. Y., Tan, T. M. C., Ong, Y. M., and Chua, K. L. (2004). BpeAB-OprB, a multidrug efflux pump in Burkholderia pseudomallei. Antimicrob. Agents Chemother. 48, 1128-1135. doi: 10.1128/AAC.48.4.1128-1135.2004

Cheng, A. C., Dance, D. A., and Currie, B. J. (2005). Bioterrorism, glanders and melioidosis. Euro. Surveill. 10, E1-E2; author reply E1-E2.

Coenye, T., Van Acker, H., Peeters, E., Sass, A., Buroni, S., Riccardi, G., et al. (2011). Molecular mechanisms of chlorhexidine tolerance in Burkholderia cenocepacia biofilms. Antimicrob. Agents Chemother. 55, 1912-1919. doi: 10.1128/AAC.01571-10

Cummings, J. E., Beaupre, A. J., Knudson, S. E., Liu, N., Yu, W., Neckles, C., et al. (2014). Substituted diphenyl ethers as a novel chemotherapeutic platform against Burkholderia pseudomallei. Antimicrob. Agents Chemother. 58, 1646-1651. doi: 10.1128/AAC.02296-13

Currie, B. J., Ward, L., and Cheng, A. C. (2010). The epidemiology and clinical spectrum of melioidosis: 540 cases from the 20 year Darwin prospective study. PLoS Negl. Trop. Dis. 4:e900. doi: 10.1371/journal.pntd.0000900

Drevinek, P., and Mahenthiralingam, E. (2010). Burkholderia cenocepacia in cystic fibrosis: epidemiology and molecular mechanisms of virulence. Clin. Microbiol. Infect. 16, 821-830. doi: 10.1111/j.1469-0691.2010.03237.x
Dubarry, N., Du, W., Lane, D., and Pasta, F. (2010). Improved electrotransformation and decreased antibiotic resistance of the cystic fibrosis pathogen Burkholderia cenocepacia strain J2315. Appl. Environ. Microbiol. 76, 1095-1102. doi: 10.1128/AEM.02123-09

Evans, K., Passador, L., Srikumar, R., Tsang, E., Nezezon, J., and Poole, K. (1998). Influence of the MexAB-OprM multidrug efflux system on quorum sensing in Pseudomonas aeruginosa. J. Bacteriol. 180, 5443-5447.

Fehlner-Gardiner, C. C., and Valvano, M. A. (2002). Cloning and characterization of the Burkholderia vietnamiensis norM gene encoding a multidrug efflux system. FEMS Microbiol. Lett. 215, 279-283. doi: 10.1111/j.15746968.2002.tb11403.x

Fujisaki, S., Ohnuma, S., Horiuchi, T., Takahashi, I., Tsukui, S., Nishimura, Y., et al. (1996). Cloning of a gene from Escherichia coli that confers resistance to fosmidomycin as a consequence of amplification. Gene 175, 83-87. doi: 10.1016/0378-1119(96)00128-X

Gamberi, T., Rocchiccioli, S., Papaleo, M., Magherini, F., Citti, L., Buroni, S., et al. (2013). RND-4 efflux transporter gene deletion in Burkholderia cenocepacia J2315: a proteomic analysis. J. Proteome Sci. Comp. Biol. 2:1. doi: 10.7243/2050-2273-2-1

Glass, M. B., Gee, J. E., Steigerwalt, A. G., Cavuoti, D., Barton, T., Hardy, R. D., et al. (2006). Pneumonia and septicemia caused by Burkholderia thailandensis in the United States. J. Clin. Microbiol. 44, 4601-4604. doi: 10.1128/JCM.01 585-06

Golini, G., Favari, F., Marchetti, F., and Fontana, R. (2004). Bacteriostatic and bactericidal activity of levofloxacin against clinical isolates from cystic fibrosis patients. Eur. J. Clin. Microbiol. Infect. Dis. 23, 798-800. doi: 10.1007/s10096004-1216-3

Grove, A. (2010). Urate-responsive MarR homologs from Burkholderia. Mol. Biosyst. 6, 2133-2142. doi: 10.1039/c0mb00086h

Guglierame, P., Pasca, M. R., De Rossi, E., Buroni, S., Arrigo, P., Manina, G., et al. (2006). Efflux pump genes of the resistance-nodulation-division family in Burkholderia cenocepacia genome. BMC Microbiol. 6:66. doi: 10.1186/14712180-6-66

Hamad, M. A., Skeldon, A. M., and Valvano, M. A. (2010). Construction of aminoglycoside-sensitive Burkholderia cenocepacia strains for use in studies of intracellular bacteria with the gentamicin protection assay. Appl. Environ. Microbiol. 76, 3170-3176. doi: 10.1128/AEM.03024-09

Hancock, R. E. W. (1998). Resistance mechanisms in Pseudomonas aeruginosa and other non-fermentative bacteria. Clin. Infect. Dis. 27(Suppl. 1), S93-S99. doi: $10.1086 / 514909$

Hayashi, S., Abe, M., Kimoto, M., Furukawa, S., and Nakazawa, T. (2000). The DsbA-DsbB disulfide bond formation system of Burkholderia cepacia is involved in the production of protease and alkaline phosphatase, motility, metal resistance, and multi-drug resistance. Microbiol. Immunol. 44, 41-50. doi: 10.1111/j.1348-0421.2000.tb01244.x

Hayden, H. S., Lim, R., Brittnacher, M. J., Sims, E. H., Ramage, E. R., Fong, C., et al. (2012). Evolution of Burkholderia pseudomallei in recurrent melioidosis. PLoS ONE 7:e36507. doi: 10.1371/journal.pone.0036507

Hemsley, C. M., Luo, J. X., Andreae, C. A., Butler, C. S., Soyer, O. S., and Titball, R. W. (2014). Bacterial drug tolerance under clinical conditions is governed by anaerobic adaptation but not anaerobic respiration. Antimicrob. Agents Chemother. 58, 5775-5783. doi: 10.1128/AAC.02793-14

Hirakata, Y., Kondo, A., Hoshino, K., Yano, H., Arai, K., Hirotani, A., et al. (2009). Efflux pump inhibitors reduce the invasiveness of Pseudomonas aeruginosa. Int. J. Antimicrob. Agents 34, 343-346. doi: 10.1016/j.ijantimicag.2009. 06.007

Holden, M. T., Seth-Smith, H. M., Crossman, L. C., Sebaihia, M., Bentley, S. D., Cerdeno-Tarraga, A. M., et al. (2009). The genome of Burkholderia cenocepacia J2315, an epidemic pathogen of cystic fibrosis patients. J. Bacteriol. 191, 261-277. doi: 10.1128/JB.01230-08

Holden, M. T. G., Titball, R. W., Peacock, S. J., Cerdeno-Tarraga, A. M., Atkins, T. P., Crossman, L. C., et al. (2004). Genomic plasticity of the causative agent of melioidosis, Burkholderia pseudomallei. Proc. Natl. Acad. Sci. U.S.A. 101, 14240-14245. doi: 10.1073/pnas.0403302101

Holland, D. J., Wesley, A., Drinkovic, D., and Currie, B. J. (2002). Cystic fibrosis and Burkholderia pseudomallei Infection: an emerging problem? Clin. Infect. Dis. 35, e138-e140. doi: 10.1086/344447

Jassem, A. N., Forbes, C. M., and Speert, D. P. (2014). Investigation of aminoglycoside resistance inducing conditions and a putative AmrAB-OprM efflux 
system in Burkholderia vietnamiensis. Ann. Clin. Microbiol. Antimicrob. 13:2. doi: 10.1186/1476-0711-13-2

Jassem, A. N., Zlosnik, J. E., Henry, D. A., Hancock, R. E., Ernst, R. K., and Speert, D. P. (2011). In vitro susceptibility of Burkholderia vietnamiensis to aminoglycosides. Antimicrob. Agents Chemother. 55, 2256-2264. doi: 10.1128/AAC.01434-10

Kang, Y., Carlson, R., Tharpe, W., and Schell, M. A. (1998). Characterization of genes involved in biosynthesis of a novel antibiotic from Burkholderia cepacia BC11 and their role in biological control of Rhizoctonia solani. Appl. Environ. Microbiol. 64, 3939-3947.

Koehler, T., Michea-Hamzehpour, M., Henze, U., Gotoh, N., Curty, L. K., and Pechere, J. C. (1997). Characterization of MexE-MexF-OprN, a positively regulated multidrug efflux system of Pseudomonas aeruginosa. Mol. Microbiol. 23, 345-354. doi: 10.1046/j.1365-2958.1997.2281594.x

Koehler, T., Van Delden, C., Kocjanic Curty, L., Hamzehpour, M. M., and Pechere, J.-C. (2001). Overexpression of the MexEF-OprN multidrug efflux system affects cell-to cell signaling in Pseudomonas aeruginosa. J. Bacteriol. 183, 52135222. doi: 10.1128/JB.183.18.5213-5222.2001

Kumar, A., Chua, K.-L., and Schweizer, H. P. (2006). Method for regulated expression of single-copy efflux pump genes in a surrogate Pseudomonas aeruginosa strain: identification of the BpeEF-OprC chloramphenicol and trimethoprim efflux pump of Burkholderia pseudomallei 1026b. Antimicrob. Agents Chemother. 50, 3460-3463. doi: 10.1128/AAC.00440-06

Kumar, A., Mayo, M., Trunck, L. A., Cheng, A. C., Currie, B. J., and Schweizer, H. P. (2008). Expression of resistance-nodulation-cell division efflux pumps in commonly used Burkholderia pseudomallei strains and clinical isolates from Northern Australia. Trans. R. Soc. Trop. Med. Hyg. 102(Suppl. 1), S145-S151. doi: 10.1016/S0035-9203(08)70032-4

Li, X. Z., Nikaido, H., and Poole, K. (1995). Role of MexA-MexB-OprM in antibiotic efflux in Pseudomonas aeruginosa. Antimicrob. Agents Chemother. 39, 1948-1953. doi: 10.1128/AAC.39.9.1948

Liu, H., Ibrahim, M., Qiu, H., Kausar, S., Ilyas, M., Cui, Z., et al. (2015). Protein profiling analyses of the outer membrane of Burkholderia cenocepacia reveal a niche-specific proteome. Microb. Ecol. 69, 75-83. doi: 10.1007/s00248-0140460-z

Liu, N., Cummings, J. E., England, K., Slayden, R. A., and Tonge, P. J. (2011). Mechanism and inhibition of the FabI enoyl-ACP reductase from Burkholderia pseudomallei. J. Antimicrob. Chemother. 66, 564-573. doi: 10.1093/jac/d kq509

Losada, L., Ronning, C. M., Deshazer, D., Woods, D., Fedorova, N., Kim, H. S., et al. (2010). Continuing evolution of Burkholderia mallei through genome reduction and large-scale rearrangements. Genome Biol. Evol. 2, 102-116. doi: $10.1093 /$ gbe/evq003

Loutet, S. A., and Valvano, M. A. (2011). Extreme antimicrobial peptide and polymyxin B resistance in the genus Burkholderia. Front. Microbiol. 2:159. doi: 10.3389/fmicb.2011.00159

Mahenthiralingam, E., Urban, T. A., and Goldberg, J. B. (2005). The multifarious, multireplicon Burkholderia cepacia complex. Nat. Rev. Microbiol. 3, 144-156. doi: $10.1038 /$ nrmicro 1085

Maravic, A., Skocibusic, M., Sprung, M., Samanic, I., Puizina, J., and Pavela-Vrancic, M. (2012). Occurrence and antibiotic susceptibility profiles of Burkholderia cepacia complex in coastal marine environment. Int. J. Environ. Health Res. 22, 531-542. doi: 10.1080/09603123.2012.6 67797

McGowan, J. E. Jr. (2006). Resistance in non-fermenting gram-negative bacteria: multidrug resistance to the maximum. Am. J. Infect. Control 34, S29-S37; discussion S64-S73.

Messiaen, A. S., Verbrugghen, T., Declerck, C., Ortmann, R., Schlitzer, M., Nelis, H., et al. (2011). Resistance of the Burkholderia cepacia complex to fosmidomycin and fosmidomycin derivatives. Int. J. Antimicrob. Agents 38, 261-264. doi: 10.1016/j.ijantimicag.2011.04.020

Mima, T., and Schweizer, H. P. (2010). The BpeAB-OprB efflux pump of Burkholderia pseudomallei $1026 \mathrm{~b}$ does not play a role in quorum sensing, virulence factor production, or extrusion of aminoglycosides but is a broadspectrum drug efflux system. Antimicrob. Agents Chemother. 54, 3113-3120. doi: 10.1128/AAC.01803-09

Mima, T., Schweizer, H. P., and Xu, Z.-Q. (2011). In vitro activity of cethromycin against Burkholderia pseudomallei and investigation of mechanism of resistance. J. Antimicrob. Chemother. 66, 73-78. doi: 10.1093/jac/dkq391
Mine, T., Morita, Y., Kataoka, A., Mizushima, T., and Tsuchiya, T. (1999). Expression in Escherichia coli of a new multidrug efflux pump, MexXY, from Pseudomonas aeruginosa. Antimicrob. Agents Chemother. 43, 415-417.

Mira, N. P., Madeira, A., Moreira, A. S., Coutinho, C. P., and Sa-Correia, I. (2011). Genomic expression analysis reveals strategies of Burkholderia cenocepacia to adapt to cystic fibrosis patients' airways and antimicrobial therapy. PLOS ONE 6:e28831. doi: 10.1371/journal.pone.0028831

Moore, R. A., Deshazer, D., Reckseidler, S., Weissman, A., and Woods, D. E. (1999). Efflux-mediated aminoglycoside and macrolide resistance in Burkholderia pseudomallei. Antimicrob. Agents Chemother. 43, 465-470.

Morita, Y., Tomida, J., and Kawamura, Y. (2012). MexXY multidrug efflux system of Pseudomonas aeruginosa. Front. Microbiol. 3:408. doi: 10.3389/fmicb.2012.00408

Nair, B. M., Cheung, K. J. Jr., Griffith, A., and Burns, J. L. (2004). Salicylate induces an antibiotic efflux pump in Burkholderia cepacia complex genomovar III (B. cenocepacia). J. Clin. Invest. 113, 464-473. doi: 10.1172/JCI200419710

Nair, B. M., Joachimiak, L. A., Chattopadhyay, S., Montano, I., and Burns, J. L. (2005). Conservation of a novel protein associated with an antibiotic efflux operon in Burkholderia cenocepacia. FEMS Microbiol. Lett. 245, 337-344. doi: 10.1016/j.femsle.2005.03.027

Nierman, W. C., Deshazer, D., Kim, H. S., Tettelin, H., Nelson, K. E., Feldblyum, T., et al. (2004). Structural flexibility in the Burkholderia mallei genome. Proc. Natl. Acad. Sci. U.S.A. 101, 14246-14251. doi: 10.1073/pnas.04033 06101

Nikaido, H., and Pages, J. M. (2012). Broad-specificity efflux pumps and their role in multidrug resistance of Gram-negative bacteria. FEMS Microbiol. Rev. 36, 340-363. doi: 10.1111/j.1574-6976.2011.00290.x

Nishino, K., and Yamaguchi, A. (2001). Analysis of a complete library of putative drug transporter genes in Escherichia coli. J. Bacteriol. 183, 5803-5812. doi: 10.1128/JB.183.20.5803-5812.2001

O'Quinn, A. L., Wiegand, E. M., and Jeddeloh, J. A. (2001). Burkholderia pseudomallei kills the nematode Caenorhabditis elegans using an endotoxinmediated paralysis. Cell Microbiol. 3, 381-393. doi: 10.1046/j.1462-5822.2001.0 0118.x

Pearson, J. P., Van Delden, C., and Iglewski, B. H. (1999). Active efflux and diffusion are involved in transport of Pseudomonas aeruginosa cell-to-cell signals. J. Bacteriol. 181, 1203-1210.

Peeters, E., Nelis, H. J., and Coenye, T. (2009). In vitro activity of ceftazidime, ciprofloxacin, meropenem, minocycline, tobramycin and trimethoprim/sulfamethoxazole against planktonic and sessile Burkholderia cepacia complex bacteria. J. Antimicrob. Chemother. 64, 801-809. doi: $10.1093 / \mathrm{jac} / \mathrm{dkp} 253$

Perrin, E., Fondi, M., Papaleo, M. C., Maida, I., Buroni, S., Pasca, M. R., et al. (2010). Exploring the HME and HAE1 efflux systems in the genus Burkholderia. BMC Evol. Biol. 10:164. doi: 10.1186/1471-2148-10-164

Podin, Y., Sarovich, D. S., Price, E. P., Kaestli, M., Mayo, M., Hii, K., et al. (2014). Burkholderia pseudomallei isolates from Sarawak, Malaysian Borneo, are predominantly susceptible to aminoglycosides and macrolides. Antimicrob. Agents Chemother. 58, 162-166. doi: 10.1128/AAC.01842-13

Podnecky, N. L., Wuthiekanun, V., Peacock, S. J., and Schweizer, H. P. (2013). The BpeEF-OprC efflux pump is responsible for widespread trimethoprim resistance in clinical and environmental Burkholderia pseudomallei isolates. Antimicrob. Agents Chemother. 57, 4381-4386. doi: 10.1128/AAC.00660-13

Poole, K. (2001). Multidrug efflux pumps and antimicrobial resistance in Pseudomonas aeruginosa and related organisms. J. Mol. Microbiol. Biotechnol. 3, 255-264.

Poole, K., Krebes, K., Mcnally, C., and Neshat, S. (1993). Multiple antibiotic resistance in Pseudomonas aeruginosa: evidence for involvement of an efflux operon. J. Bacteriol. 175, 7363-7372.

Price, E. P., Sarovich, D. S., Mayo, M., Tuanyok, A., Drees, K. P., Kaestli, M., et al. (2013). Within-host evolution of Burkholderia pseudomallei over a twelve-year chronic carriage infection. MBio 4:4. doi: 10.1128/mBio.00388-13

Propst, K. L. (2011). The Analysis of Burkholderia pseudomallei Virulence and Efficacy of Potential Therapeutics. Ph.D. Dissertation, Colorado State University, Fort Collins, CO.

Propst, K. L., Mima, T., Choi, K. H., Dow, S. W., and Schweizer, H. P. (2010). A Burkholderia pseudomallei $\triangle$ purM mutant is avirulent in immune competent and immune deficient animals: candidate strain for exclusion from select agent lists. Infect. Immun. 78, 3136-3143. doi: 10.1128/IAI.01313-09 
Rajendran, R., Quinn, R. F., Murray, C., Mcculloch, E., Williams, C., and Ramage, G. (2010). Efflux pumps may play a role in tigecycline resistance in Burkholderia species. Int. J. Antimicrob. Agents 36, 151-154. doi: 10.1016/j.ijantimicag.2010.03.009

Rushton, L., Sass, A., Baldwin, A., Dowson, C. G., Donoghue, D., and Mahenthiralingam, E. (2013). Key role for efflux in the preservative susceptibility and adaptive resistance of Burkholderia cepacia complex bacteria. Antimicrob. Agents Chemother. 57, 2972-2980. doi: 10.1128/AAC.00140-13

Sass, A., Marchbank, A., Tullis, E., Lipuma, J. J., and Mahenthiralingam, E. (2011). Spontaneous and evolutionary changes in the antibiotic resistance of Burkholderia cenocepacia observed by global gene expression analysis. BMC Genomics 12:373. doi: 10.1186/1471-2164-12-373

Schell, M. A., Zhao, P., and Wells, L. (2011). Outer membrane proteome of Burkholderia pseudomallei and Burkholderia mallei from diverse growth conditions. J. Proteome Res. 10, 2417-2424. doi: 10.1021/pr1012398

Schulin, T., and Steinmetz, I. (2001). Chronic melioidosis in a patient with cystic fibrosis. J. Clin. Microbiol. 39, 1676-1677. doi: 10.1128/JCM.39.4.16761677.2001

Schweizer, H. (2012a). "Mechanisms of Burkholderia pseudomallei antimicrobial resistance," in Melioidosis - A Century of Observation and Research, ed. N. Ketheesan (Amsterdam: Elsevier), 229-238.

Schweizer, H. P. (2012b). Mechanisms of antibiotic resistance in Burkholderia pseudomallei: implications for treatment of melioidosis. Future Microbiol. 7, 1389-1399. doi: $10.2217 /$ fmb. 12.116

Schweizer, H. P. (2012c). When it comes to drug discovery not all Gram-negative bacterial biodefence pathogens are created equal: Burkholderia pseudomalle $i$ is different. Microb. Biotechnol. 5, 581-583. doi: 10.1111/j.1751-7915.2012.00334.x

Scoffone, V. C., Spadaro, F., Udine, C., Makarov, V., Fondi, M., Fani, R., et al. (2014). Mechanism of resistance to an antitubercular 2-thiopyridine derivative that is also active against Burkholderia cenocepacia. Antimicrob. Agents Chemother. 58, 2415-2417. doi: 10.1128/AAC.02438-13

Scott, A. E., Laws, T. R., D'elia, R. V., Stokes, M. G., Nandi, T., Williamson, E. D., et al. (2013). Protection against experimental melioidosis following immunization with live Burkholderia thailandensis expressing a manno-heptose capsule. Clin. Vaccine Immunol. 20, 1041-1047. doi: 10.1128/CVI.00113-13

Sim, B. M., Chantratita, N., Ooi, W. F., Nandi, T., Tewhey, R., Wuthiekanun, V., et al. (2010). Genomic acquisition of a capsular polysaccharide virulence cluster by non-pathogenic Burkholderia isolates. Genome Biol. 11:R89. doi: 10.1186/gb-2010-11-8-r89

Simpson, A. J., Newton, P. N., Chierakul, W., Chaowagul, W., and White, N. J. (2003). Diabetes mellitus, insulin, and melioidosis in Thailand. Clin. Infect. Dis. 36, e71-e72. doi: 10.1086/367861

Sobel, M. L., Mckay, G. A., and Poole, K. (2003). Contribution of the MexXY multidrug transporter to aminoglycoside resistance in Pseudomonas aeruginosa clinical isolates. Antimicrob. Agents Chemother. 47, 3202-3207. doi: 10.1128/AAC.47.10.3202-3207.2003

Song, H., Hwang, J., Yi, H., Ulrich, R. L., Yu, Y., Nierman, W. C., et al. (2010). The early stage of bacterial genome-reductive evolution in the host. PLoS Pathog. 6:e1000922. doi: 10.1371/journal.ppat.1000922

Tegos, G. P., Haynes, M. K., and Schweizer, H. P. (2012). Dissecting novel virulent determinants in the Burkholderia cepacia complex. Virulence 3, 234-237. doi: 10.4161/viru.19844

Teng, M., Hilgers, M. T., Cunningham, M. L., Borchardt, A., Locke, J. B., Abraham, S., et al. (2013). Identification of bacteria-selective threonyl-tRNA synthetase substrate inhibitors by structure-based design. J. Med. Chem. 56, 1748-1760. doi: 10.1021/jm301756m

Tian, Z. X., Mac Aogain, M., O'connor, H. F., Fargier, E., Mooij, M. J., Adams, C., et al. (2009). MexT modulates virulence determinants in Pseudomonas aeruginosa independent of the MexEF-OprN efflux pump. Microb. Pathog. 47, 237-241. doi: 10.1016/j.micpath.2009.08.003
Trunck, L. A., Propst, K. L., Wuthiekanun, V., Tuanyok, A., Beckstrom-Sternberg, S. M., Beckstrom-Sternberg, J. S., et al. (2009). Molecular basis of rare aminoglycoside susceptibility and pathogenesis of Burkholderia pseudomallei clinical isolates from Thailand. PLoS Negl. Trop. Dis. 3:e0000519. doi: 10.1371/journal.pntd.0000519

Tseng, S. P., Tsai, W. C., Liang, C. Y., Lin, Y. S., Huang, J. W., Chang, C. Y., et al. (2014). The contribution of antibiotic resistance mechanisms in clinical Burkholderia cepacia complex isolates: an emphasis on efflux pump activity. PLoS ONE 9:e104986. doi: 10.1371/journal.pone.01 04986

Vandamme, P., and Dawyndt, P. (2011). Classification and identification of the Burkholderia cepacia complex: Past, present and future. Syst. Appl. Microbiol. 34, 87-95. doi: 10.1016/j.syapm.2010.10.002

Vanlaere, E., Baldwin, A., Gevers, D., Henry, D., De Brandt, E., Lipuma, J. J., et al. (2009). Taxon K, a complex within the Burkholderia cepacia complex, comprises at least two novel species, Burkholderia contaminans sp. nov. and Burkholderia lata sp. nov. Int. J. Syst. Evol. Microbiol. 59, 102-111. doi: 10.1099/ijs.0.00 1123-0

Viktorov, D. V., Zakharova, I. B., Podshivalova, M. V., Kalinkina, E. V., Merinova, O. A., Ageeva, N. P., et al. (2008). High-level resistance to fluoroquinolones and cephalosporins in Burkholderia pseudomallei and closely related species. Trans. R. Soc. Trop. Med. Hyg. 102(Suppl. 1), S103-S110. doi: 10.1016/S00359203(08)70025-7

Vora, S. K. (2002). Sherlock Holmes and a biological weapon. J. R. Soc. Med. 95 , 101-103. doi: 10.1258/jrsm.95.2.101

Whitlock, G. C., Estes, D. M., and Torres, A. G. (2007). Glanders: off to the races with Burkholderia mallei. FEMS Microbiol. Lett. 277, 115-122. doi: 10.1111/j.1574-6968.2007.00949.x

Wiersinga, W. J., Birnie, E., Weehuizen, T. A., Alabi, A. S., Huson, M. A., In 't Veld, R. A., et al. (2015). Clinical, environmental, and serologic surveillance studies of melioidosis in Gabon, 2012-2013. Emerg. Infect. Dis. 21, 40-47. doi: 10.3201/eid2101.140762

Wiersinga, W. J., Currie, B. J., and Peacock, S. J. (2012). Melioidosis. N. Engl. J. Med. 367, 1035-1044. doi: 10.1056/NEJMra1204699

Wiersinga, W. J., Van Der Poll, T., White, N. J., Day, N. P., and Peacock, S. J. (2006). Melioidosis: insights into the pathogenicity of Burkholderia pseudomallei. Nat. Rev. Microbiol. 4, 272-282. doi: 10.1038/nrmicro1385

Wigfield, S. M., Rigg, G. P., Kavari, M., Webb, A. K., Matthews, R. C., and Burnie, J. P. (2002). Identification of an immunodominant drug efflux pump in Burkholderia cepacia. J. Antimicrob. Chemother. 49, 619-624. doi: 10.1093/jac/49.4.619

Winsor, G. L., Khaira, B., Van Rossum, T., Lo, R., Whiteside, M. D., and Brinkman, F. S. L. (2008). The Burkholderia genome database: facilitating flexible queries and comparative analyses. Bioinformatics 24, 2803-2804. doi: 10.1093/bioinformatics/btn 524

Zhang, L., Li, X. Z., and Poole, K. (2001). Fluoroquinolone susceptibilities of effluxmediated multidrug-resistant Pseudomonas aeruginosa, Stenotrophomonas maltophilia and Burkholderia cepacia. J. Antimicrob. Chemother. 48, 549-552. doi: $10.1093 / \mathrm{jac} / 48.4 .549$

Conflict of Interest Statement: The authors declare that the research was conducted in the absence of any commercial or financial relationships that could be construed as a potential conflict of interest.

Copyright (C) 2015 Podnecky, Rhodes and Schweizer. This is an open-access article distributed under the terms of the Creative Commons Attribution License (CC BY). The use, distribution or reproduction in other forums is permitted, provided the original author(s) or licensor are credited and that the original publication in this journal is cited, in accordance with accepted academic practice. No use, distribution or reproduction is permitted which does not comply with these terms. 